\title{
The use of information technology in training human
}

resources:

\author{
An E-learning case study \\ José L. Gascó, Juan Llopis ${ }^{1}$ \& M. Reyes González \\ Department of Firm Organization, University of Alicante, 03080 San \\ Vicente (Alicante), Spain
}

\begin{abstract}
This paper deals with the influence of IT (Information Technology) in HRM (Human Resources Management) and more specifically on training policy, by means of the experience in this field of a Spanish telecommunications firm, Telefonica. For this, we will go into more depth about the characteristics of the training model designed by this firm to face new environments, the technologies used, the actions which have had most impact, the disadvantages and the success factors that have been detected trying to grow an Elearning company. The study of this case has facilitated the identification of the following successful factors in a training policy: flexibility in time management for training; active participation by trainers; the establishment of control mechanisms that guarantee that the training really occurs; the creation of quality content; the promotion of interactive elements among trainers, among students and with each other; the use of standardised and developed technologies and the gradual implementation of these experiences. As regards, objectives for the future emphasis, in the company analysed is on maintaining progress in the use of E-learning as a way of adapting the training process to the new E-business culture.
\end{abstract}

Keywords: E-learning; HR and IT; Training; Case study

\footnotetext{
${ }^{1}$ Corresponding autor. Tel: 34-965903607; fax: 34-965903606.

E-mail address: juan.llopis@ua.es
} 


\section{The use of information technology in training human resources:}

\section{An E-learning case study}

\section{Human resources management and employability}

The Human Resources (HR from now on) function currently finds itself in an unprecedented situation. According to some authors, this function has already experienced numerous changes and has gone a long way. This is obviously true and so far, it has been adapting to the changes occurring in its environment, mainly because those changes were slow and, to some extent, foreseeable. However, the opposite happens now; the speed at which changes are occurring is increasingly high and the course the business world is taking is also more and more unforeseeable. This is why HR managers will not only have to make mere adaptations, but face true organisational transformations.

Within such a context, we think survival will only be possible for those firms which understand that their HR managers cannot have a secondary role, from the professional point of view, in the strategic decision-making process and must gain enough relevance within the firm's business policy, which will made them able to face the organisations' increasingly difficult challenges with managerial-type decisions. They will achieve this relevance when the HR function: 
- Is a true strategic partner of the top management, but at the same time, correctly performs its role of spokesman-defender of employees' interests (Ehrlich, 1997 and Ellig, 1997).

- Has outsourced numerous functions, but is better equipped in qualitative terms (Christensen, 1997; and Gómez Mejía, Balkin \& Cardy, 2001).

- Without forgetting internal economic aspects, is strongly involved in finding solutions to the economic and social problems of the society that surrounds it; that is to say, when it co-ordinates internal affairs with its economic and social environment (Jacoby, 1985; Kochan, 1997 and Lobel, 1997).

- Focuses on helping the firm to accomplish its aims and is able to show clearly how this function contributes to the organisation's profits (Fonda \& Buckton, 1995 and Ulrich, 1997).

- Catalyses change in its organisations; in other words, it must be proactive, identify opportunities for change and improvement, convert ideas into tangible concepts and make them come true. The reason is very simple: no organisational change can take place without a change of behaviours.

- Is capable of guaranteeing collaborators' employability, without compromising firm flexibility, since, in future, people will form part of organisations for their ability to do things and not for what they represent or for the knowledge they acquired in the past. 
This last challenge, achieving collaborators' employability, is one of the most important in our point of view. Continuity in the work relationship and collaborator motivation are closely related concepts, the second being nearly impossible without the first. Nevertheless, hardly any firm can guarantee employment for life at present. However, in order to achieve employees' commitment and motivation, managers must commit themselves to offering jobs and opportunities for work and professional development that allow employees to somehow maintain their contribution to the labour market in terms of value and, if managers have to do without those employees at any given moment, they must leave them in a situation in which their incorporation to another job is easy.

In order to achieve this employability level for collaborators, HR management policies must be designed that focus on changing attitudes and accepting change, making employees responsible for their own achievements. The challenge consists in developing a technical system that makes it easy for employees to solve most of the problems related to their job, making them able to sort out those problems that they have so far been unable to solve and have had to refer back to higher hierarchical levels (Townsend \& Hendrickson, 1996).

If we pay attention to our model of society, more and more importance and responsibility are being given to the client, the investor, the student or the citizen. We are evolving towards the society of self-service; sentences like "help yourself", "do it yourself" 
are omnipresent in numerous hypermarkets, petrol stations, banks, restaurants or cafés. In the HR area, this idea finds a simile in the possibility employees have of managing their own information, being responsible for its updating and having direct access to it, no matter if that information refers to training, performance assessment, transfers or any other aspect that affects employees. Some call this idea employee self-service. This concept is perhaps older than the Internet/Intranet, and even more than the PC; but these technologies undoubtedly facilitate the development of this idea. The logic underpinning this term centres on training employees so that they can acquire responsibility about matters that have traditionally been centralised in a staff, bureaucratic department. Transferring responsibility to the employee himself actually means relocating the individual within the firm, favouring his expansion and motivation and contributing to his employability.

In this paper, we are going to analyse how information and communication technologies (ICT from now on) can encourage this process, while, at the same time, we describe the efforts a large Spanish firm has made in this field.

\section{Intranets in human resources departments}

Some suitable technological developments are required to implement the idea of employee self- service we have mentioned in 
the previous paragraph. Among them stand out Intranets. The Intranet is a powerful tool which can, if correctly used, encourage communication and collaboration in the firm, tidy up procedures and provide its staff with permanently updated information, even if the staff are scattered all over the world. The reasons that have led large firms to install Intranets in their HR departments are not only linked with the transfer of $\mathrm{HR}$ data management to employees and functional managers themselves. A review of the specialised literature (Alberston, 1999; Ammenheuser, 2000; Burzawa, 1997; Geoffrey, 1997; Kristen, 1997; Rogers, 2000 and Stedman, 1999) gives us reasons like the following:

- Collecting, communicating and sharing information with employees.

- Automating access and administration to databases.

- Simplifying the distribution of complex information, presenting it much more thoroughly and graphically.

- Building an infrastructure which increases people's effectiveness in the accomplishment of their aims.

- Helping managers to identify experts on the basis of such criteria as skills, knowledge, experience and place of residence.

- Installing best practice files, which spares people who consult them from having to start each project from scratch and makes it possible to locate experts in one particular field within the firm. 
- Creating on-line forums which allow an employee that is assigned to a project in a specific sector to connect with one forum and seek advice from the firm's experts.

The same as reasons are diverse, it is also easy to find arguments in the specialised literature about the numerous advantages derived from the building of an Intranet from the point of view of HR management (Blair, 2000; Dawson, 1998; Hirschfield \& Currie, 1997; Perussina, 1998; Perussina, 2000; Quinn, 2000). Among hard benefits, we can highlight the following: paper and edition savings; updating easiness; reductions in the number of answers to employees' repetitive questions; the possibility of consulting, at any given place and time, a wide range of reference materials (training packages, policy and procedure manuals, announcements of jobs and important events, statements and memoranda).

Soft benefits are, due to their own nature, intangible and therefore, difficult to quantify. They generally have to do with the improvement of the service offered by the HR function and with the fact that more time can be dedicated to the most important activities, which, in turn, improves the perception that both management and employees have of this function. Furthermore, other advantages can be highlighted such as the promotion of innovation, the flexibility of structures and the possibility of individualising labour relations.

Nevertheless, what many experts forget, although it is perhaps the most important thing, is the fact that an Intranet makes it possible to relocate the individual within the firm and increases his 
expansion and motivation levels, thus contributing to his employability. Intranet-type architectures contain tools that favour this evolution, as they give everybody access to the firm's common asset: information. In this situation, the individual employability of each firm member will be a function of the use each one of them makes of the information that the firm makes available to everybody.

However, before building an Intranet, any firm should have a clear idea of its future use. If an Intranet is developed only because it is in fashion, the Intranet runs the risk of being eliminated for not providing added value to the organisation. The main value will appear when people change the way in which important organisational procedures are carried out. A successful Intranet project must be linked with the analysis of existing procedures in order to determine where it is possible to obtain the best profits. When this has become clear for an organisation, it will have to solve other more technical or operational aspects: changes in the organisational structure; hardware, software and security system requirements; responsibility assignation mechanisms, both regarding the capacity to publish and in relation to contents and updates; identification of potential users along with their levels of interest; methods to detect specific information needs; systems of solutions to conflicts derived from the excessive expectations the project can generate... (Barry, 1998; Elswick, 2000; Esplin, 1998; Frazee, 1998; Hills, 1996; Holtz, 1997; Lapointe, 1998; Martin, 1998; Martin, 1999; Meuse, 1999; Roberts, 1998 and Starcke, 1997). 
Finally, we must not forget that these approaches do not represent a panacea. Technology on its own will not suffice for experts to share their knowledge with others. Neither will it make employees not keen on acquiring knowledge sit in front of the keyboard and start surfing and doing research. However, if an organisation already has the aspirations, the skills and the attention focused on knowledge, technology will facilitate the access to that knowledge, and pave the way for the suitable knowledge to reach the right person in the right moment.

The above-mentioned approaches based on ICT introduction in HR departments are not yet a frequent practice in most Spanish firms, since the introduction of these technologies requires a technological development that many countries have not reached. Without wanting to underestimate other firms' efforts, we think that Telefonica has been one of the pioneers in the introduction of ICTbased HR management. Some 33,000 of its employees had access to its Intranet portal E Domus at the beginning of 2001. To the over 100 on-line courses that can be consulted on the Intranet, we have to add news, interviews, reports, trade union's board, vacancies, directory and even a monthly contest with the best initiatives. For these reasons, analysing this firm's specific experience can be of great help to understand the real possibilities Intranets offer in the HR area. More specifically we will focus exclusively on one aspect of HR management: training. 


\section{Telefonica's training model for the digital age}

When we talk about Telefonica, we are referring to the leading telecommunications operator in the Spanish and Portugues-speaking world, where it has a potential market that exceeds the figure of 550 million people, 65 million of whom are clients. It is Spain's first multinational in terms of market capitalisation and one of the world's largest telecommunications firms. Through subsidiaries in Latin America, Europe, United States, Africa and Asia, Telefonica supplies services in 41 countries. It has over a million direct shareholders. Its shares are listed on the Spanish electronic stock markets (Madrid, Barcelona, Bilbao and Valencia) as well as in the Stock Exchanges of London, Paris, Frankfurt, Tokyo, New York, Lima, Buenos Aires and Sao Paulo and in London's SEAQ International.

This firm has recently undertaken a deep revision of many usual training schemes. One of the first goals Telefonica set itself was the development of a new attitude towards all that concerns training policy. The aim was to go back to the very foundations of that policy for its collaborators to have the chance to develop their skills and competencies, thus growing in accordance with the permanent qualification revolution that accompanies ICTs and, consequently, guaranteeing their employability levels.

In this search for higher employability levels for its employees, Telefonica's training policy has focused on what they have called cooperative learning. The basic aspects in this concept are the following: 
- Interactivity: the participation and interaction of all participants is the key element. The idea is to create permanent learning platforms which are totally connected with work, considering training not as a break in the performance of the activity or a holiday circumstance, but as another form of work. The motto is "learning by doing".

- Facilitation: the trainer is not the only owner of all the information, but someone who facilitates interaction between participants and who simultaneously learns from this as a result of the multidirectional collaboration process.

- Accessibility: the learning systems used allow students (employees) to have access to training contents and tutorials without having to go to specific centres or stick to fixed timetables.

- Adaptability: Training programmes are perfectly adapted to the real, specific needs and demands of each group of employees that is trained.

- Flexibility: Since needs are changing, an agile, quick response that guarantees the acquisition of the necessary knowledge when that knowledge is actually needed (just in time) becomes essential.

- Employability: The objective sought is to train students (employees) so that they can react in front of all sorts of changes and overcome the subsequent difficulties maintaining employment possibilities. 
In order to arrive at this new approach to training policy, Telefonica has had to change several paradigms that had long been immovable in the firm:

- The role of students, who from being mere participants have come to be starring characters from whom a greater motivation, commitment and involvement are demanded.

- The position of trainers, who from being the exclusive owners of a set of knowledge and wisdom have had to assume the role of facilitators and start to see the student as someone who also helps them to learn.

A change like this could not be carried out by decree, so the process has been and continues to be the opposite: involving everybody and making them party to the project. As regards the formative methodology, Telefonica has made a strong bet on teletraining. It has implemented a distance training system which, through the combination of various pedagogic techniques, uses ICTs and introduces maximum geographical and time flexibility as distinct key elements with respect to other knowledge teaching systems.

This model seeks a double objective. On the one hand, it wants workers to acquire the skills required to perform their tasks with professionalism in the context of competitiveness that governs the world of telecommunications, thus guaranteeing their employability. On the other hand, it seeks to give the firm's clients the chance to familiarise with the goods and services supplied by that firm, with the purpose of improving its commercial performance. Therefore, the 
firm not only has to ensure the provision of the necessary technical knowledge but also manage to keep its leading position in the telecommunications sector through the use of this formative model.

The training model designed by Telefonica to accomplish these aims can be described as follows:

- Integrated into the strategic plan. The objectives set in the model are those of the organisation it serves and the model seeks the total accomplishment of those objectives. It especially focuses in helping Telefonica's human resources to acquire the necessary skills.

- Oriented towards management. The people that are entirely devoted to training do not teach courses but mainly organise and arrange them leaving the task of teaching in the hands of the teachers-collaborators, of whom there are around 25,500.

- Based on the teacher-collaborator. This option becomes compulsory, amongst other reasons, because the knowledge of technologies and work processes lies in the organisation's staff, while the specific skills are logically to be found in each business. This is why businesses must assume this model with the corresponding implications. Teachers' participation is always voluntary and their pay is that stipulated in the collective bargaining process.

- Based on internal means. The training department mainly uses internal resources to provide itself with the materials involved in each training action: the premises where it takes place, the 
technological equipment used and, of course, the documents that accompany each didactic activity.

- Focused on the demand. The training management translates into formative language (courses, pedagogic objectives, programmes, ...) the needs expressed by business areas and uses them to build the training offer that is presented in the Catálogo de Recursos Recomendados (Recommended Resources Catalogue) every year.

- Supported by assessment. The follow-up of the training given materialises in different types of assessment (summative, formative, longitudinal) performed through different computerbased procedures, which makes it possible to obtain results instantaneously. The systems used are EVATEL, suitable to satisfy the student's satisfaction level, and EVALONG, which evaluates the usefulness of training actions.

- Integrated into the collective bargaining process. Collective bargaining agreements contain abundant doctrine on training and regulate the participation of committees and trade unions in the formative system. There is a Comisión Central de Formación (Central Training Commission) and territorial commissions, all of them in a situation of parity, in which very important aspects of the training system are debated upon and negotiated.

- Participative. Employees negotiate with their immediate superior the training scheme for the following year, taking into 
account the profiles needed for an ideal job performance and also considering workers' operational knowledge as well as other types of knowledge that have to do with their personal aspirations. The possibility of improving worker employability can also act as a motivating element in this context.

\section{The role of technologies in the evolution of Telefonica's training model}

The formative model we have just described is strongly dependent on ICTs. However, it is difficult to determine when Telefonica finally bets on ICTs for the design and management of its training policy. If we analyse the firm's recent history, we can distinguish five different types of training according to the form of communication between teachers and students.

1. Asynchronous technologies. Those in which communication is recorded. There is no real-time communication between the information generator and the information receiver. The time flexibility that characterises them has made them be of preferential use in many firms. The most representative technologies in this group are: contents (CBTs and WBTs), electronic mail, discussion groups and debate forums. The first attempts to introduce them in Telefonica date back to 1993, when educational contents started to be widely distributed by means of diskettes. 
2. Synchronous technologies. Those in which communication takes place in real time. The most representative ones are: telephone, electronic blackboard, instant messaging services and videoconferencing. The year 1995 can be considered as the date in which Telefonica made its definitive bet on these technologies. It was then that the firm created the first teletraining classrooms, located in the main work centres, along with the figure of the teletutor, available by phone and Email.

3. Learning Management Tools. Quite widespread so far, but likely to be replaced by other more complex tools. These are tools used to carry out training/learning management: they make it possible to create and maintain a catalogue of courses, they facilitate student enrolment and teacher assignation processes, they manage the means to be used, the orders of formative materials, the marks, ... In general, these tools are used not to give training but to organise how it is given. The introduction of these tools dates back to 1992, but then they were only focused on the management of presential (face-toface) training courses.

4. Author's tools. They are tools used in the creation of contents and not in the management of training itself. The most widespread ones are Microsoft Frontpage, Dreamweaver, Macromedia Authorware, ToolBook II, ... 
5. Learning Management Systems. These type of products incorporate, in an integrated way, some of the abovementioned functionalities. These are systems that combine the provision and management of training in a single product. They usually have two groups of functionalities, those that have to do with administrative functions (student management, building of formative offers, student follow-up, ...) and those related to the provision of training (access to formative contents, tutorial services, communications services, ...). In 1997, Telefonica established the first version of these systems, known as Red teledidáctica (RTD) -Teledidactic Network- and based on the Global Teach V1.0 platform. However, it is only from 1999 onwards that its use as the main internal training tool became widespread. This service has been updated with new versions of the platform at an approximate rate of one version per year, and is currently used with quite a lot of success.

Telefonica's years of experience in this field show that it is not a question of choosing a particular technology; in fact, it is wiser to use different systems or, at least, different techniques and supports for its various types of formative actions. When it comes to deciding what technology to use for providing a specific formative action, it is recommended to bear in mind some of the following general factors: a) Training objectives and student needs. b) Period of validity for the knowledge to be transmitted. c) Type of formative sessions to be 
followed and frequency of interactions with students. d) Whether it is a complement or a total replacement of a presential formative action. e) Whether formative materials to be provided can be reused or have to be redesigned. f) The number of students the formative action is addressed to. g) Cost of the means and resources required, including software, computers and connection costs. h) Student predisposition to use these technologies. i) Level of trust in the technology provider and its technical support. j) The technology and communications infrastructure existing in the organisation.

\section{Telefonica's current RTD (red teledidáctica or teledidactic network)}

As we have explained in the previous section, Telefonica opted for a Learning Management System that it called Red Teledidáctica, which was the result of cooperation with two other European operators -Deutsche Telekom y Swisscom- within the framework of the Global Teach Association. The RTD is not really a network at present. It is currently nothing but one of the different services available in the corporate Intranet. The RTD is a teletraining service that Telefonica's training management makes available to all the employees so that, to meet the firm's needs or out of a personal interest of employees themselves, they can complete, enlarge or update their training in various aspects related to business activity. 
The essential objective sought with the building of the RTD was to improve employees' employability through three learning modalities:

- Self-study through free access to the net. It consists in a wide catalogue of computer- assisted teaching courses in which employees can freely choose the ones they are interested in, install them in their computer and study them when they want to and at the pace they set themselves. Among the range of courses available stand out the ones covering the latest ICTs, this being an aspect that is closely related to employees' involvement in the increase of their employability level.

- Tutorised courses in the RTD. These courses count on the presence of a teacher/tutor who provides students with learning support, orientation and supervision during their learning. In accordance with the stipulations of the collective bargaining agreement, if the tutor assesses them positively, students will be given curricular recognition in the employee's formative report. The duration of these courses is less than 100 hours.

- Telfonet, videoconferencing network. Formative or informative sessions on current topics of interest are offered through the videoconferencing room network. These sessions are taught by outstanding professionals and experts in the different fields, and students attending these courses have the chance to ask speakers questions live from their respective rooms. 
The formative activity carried out in the RTD during 2001 has had impressive results, as the number of RTD users has trebled in just one year, which means that $18 \%$ of the training has been carried out using this service. The number of employees enrolled at present is 22,000 . The use of this methodology is fully integrated into the firm's training schemes, which has already available over 80 courses on 10 different thematic areas. Four actions stand out among those that have had the greatest impact on the RTD: the Annual Training Plan, the Materials and Resources Catalogue, the Relocation Special Programme and the Entry and Promotion Programme.

- The Annual Training Plan mainly includes formal training. It is carried out through presential teaching (traditional courses) and distance courses in which diverse technologies are used.

- The Materials and Resources Catalogue is an extensive collection of documents meant to be a complement to teachers' formative function and a self-learning tool. The bibliographical collections include reference works, monographs, internal publications, reviews, videos, ... As regards topics, the Catalogue is specialised in HR and business training. However, it covers other support areas: business management and administration, computers, telecommunications policy, labour relations and languages.

- The Relocation Special Programme combines the learning and training mechanisms, making it easier for employees to acquire the skills required to be successful at a different job from the 
one they used to perform. It is addressed to employees of categories living in the provinces (states) and localities where the available resources are defined. The programme uses both face-to-face and distance teaching methods through teletraining.

- The Entry and Promotion Programme is designed for employees that have recently joined the firm or those that move to other labour groups. Its aim is to guarantee correct adaptation to the working position.

In addition to the above, the RTD is used to provide specific virtual environments for the development of career plans and other strategic programmes in which a mixed methodology that combines E-learning and presential classes is applied. It is a distance teaching/learning service that the firm puts at the disposal of all employees who voluntarily wish to complete or enlarge learning mechanisms for matters related to business activity outside their working day.

At present, it has experienced an important qualitative change and has come to be known as $\boldsymbol{a}+$. It offers employees the possibility of following from their homes through ADSL technology those courses in the formative catalogue that they find interesting, even if those courses are not directly related to their current job.

\section{Conclusions}


The results of the actions undertaken so far in the design of new training models are encouraging. According to managers responsible for these new designs, the following advantages have generally been detected: accessibility at any time and place; the preparation of 'à-la-carte' training menus adapted to the profiles of each group of students; access to a large set of teaching materials that are unavailable under normal circumstances; the simulation of situations that are very difficult or risky in real life; and the fact that they are based on the currently universally applied ICTs.

But, in addition to that, these new formative designs provide additional advantages for a firm like Telefonica that forms part of a group that is spread across several countries: the development within the firm of a multinational approach to training, cost optimisation as a result of sharing the technological platform and the contents and finally, the consolidation of the group's technological and corporate culture. This materialises in the provision of a powerful, easy-to-access, user-friendly cultural tool to all Telefonica employees as is the wide range of formative offers related to their current job or in the possibility for employees to increase their employability level.

As regards the setbacks that have been found and which, according to Telefonica, can delay its implementation in other firms, we must highlight the following: initial investment and permanent equipment update; not very homogeneous and still not 
consolidated technology; limited technological culture in both trainers and trainees along with lack of handling experience; greater effort and dedication on the part of tutors; few purposebuilt contents; need for self-motivation in trainees -experience shows that not everybody is successful at self-learning; a special commitment is required- and finally problems related to intellectual property.

The experience that Telefonica has accumulated during the last few years in the implementation of ICT-based training programmes has allowed this firm to identify the following success factors: flexibility in the management of learning times; trainers' active participation; the development of control mechanisms that ensure training effectiveness; the preparation of high-quality contents; the reinforcement of mutual interaction elements between trainers, between students and between both; the use of standardised technologies and the gradual introduction of these experiences.

Nowadays, Telefonica has as its aim to keep moving forward in the use of E-learning as a way to adapt the formative process to the new E-business culture. In this sense, the firm is permanently working on the implementation of new functionalities for the platform which will provide services that, apart from being even more sophisticated, can be integrated with other HR management corporate applications: strengthening of synchronous training and collaborative learning, module-based design of educational contents and formative actions, more decentralised service administration, 
new support services for tutors, ... As for contents, they are still being structured with the purpose of finding an integrated training model that combines collaborative and experiential learning through specific formative itineraries.

\section{References}

Albertson, D. (1999). Life support. Employee Benefit News, 13, 3436.

Ammenheuser, M. (2000). ABN AMRO profiting from self-service HR system. Bank Systems and Technology, 37(12), 31-32.

Barry, T. (1998). Flex on the Intranet. Case study: BBN Corporation. Compensation and Benefits Management, 14(2), 29-34.

Blair, L. (2000). Employee self-service research documents ROI. Employee Benefit News, 14(10), 23-25.

Burzawa, S. (1997). Intranet streamlines benefits administration and communications at Apple Computer. Employee Benefit Plan Review, 51(8), 43-45.

Christensen, R. (1997). Where is HR? Human Resource Management, 36(1), 81-84.

Dawson, S. J. (1998). Closing the gap: Lowering program costs and increasing perceived value with employee self-service. Compensation and Benefits Management, 14(2), 59-63.

Ehrlich, C. J. (1997). Human resource management: A changing script for a changing world. Human Resource Management, 36 (1), 85-89. 
Ellig, B. R. (1997). Is the human resource function neglecting the employees? Human Resource Management, 36 (1), 91-95.

Elswick, J. (2000). Successful employee self-service programs emphasize communication, not technology. Employee Benefit News, 14 (2), 21-23.

Esplin, K. (1998). 8 important issues to consider before building an Intranet. The International Association for Human Resource Information Management, httpp://www.ihrim.com.

Fonda, N. \& Buckton, K. (1995). Reviewing the personnel function. London: Institute of Personnel and Development.

Frazee, V. (1998). Assemble a project team to launch your ESS system. Workforce, 77(7), 71-72.

Geoffrey, J. (1997). IT helps HR become strategic. Datamation, 43(4), 110-115.

Gómez-Mejía, L. R., Balkin, D. B. \& Cardy, T. L. (2001). Managing human resources (third edition). Englewood Cliffs, NJ: Prentice Hall.

Hills, M. (1996). Intranet business strategies and Intranet as groupware. New York: John Wiley \& Sons.

Hirschfield, R. \& Currie, C. (1997). Shared service centres and employee self-service: Concepts are often confused, but not confusing. Employee Benefit Plan Review, 51(8), 38-41.

Holtz, S. (1997). Strategizing a human resources presence on the Intranet. Compensation and Benefits Management, 13(4), 3138. 
Jacoby, S. (1985). Employing bureaucracies. New York: Columbia University Press.

Kochan, T. (1997). Rebalancing the role of human resources. Human Resource Management, 36(1), 121-128.

Kristen, B. (1997). HR departments get new look to better serve employees. Business Journal Serving San Jose and Silicon Valley, 15(27), 49-51.

Lapointe, J. R. (1997). A method for selecting the right employee selfservice solutions. HR Magazine, 42(8), 37-44.

Lapointe, J. R. (1998). Seven steps to successful ESS. HR Focus, 75(4), 13-15.

Lobel, S. (1997). In praise of the soft stuff: A vision for human resource leadership. Human Resource Management, 36(1), 3539.

Martin, A. (1999). Survey finds dramatic ROI for HR self-service. Employee Benefit News, 13(7), 21-24.

Martin, A. (1998). Human resources self-service: One year later. The International Association for Human Resource Information Management, httpp://www.ihrim.com

Meuse, D. (1999). Making employee self-service work for employees and your company. Benefits Quarterly, 15(3), 18-24.

Perussina, R. D. (1998). Fire that elusive somebody in HR by redefining ESS. Employee Benefits Journal, 23(4), 28-32. 
Perussina, R. D. (2000). Employee self-service enables employees to leverage benefits and become self-sufficient. Employee Benefits Journal, 25(2), 15-18.

Quinn, R. D. (2000). At the Water's edge. Employee Benefit News, 14(13), 35-39.

Roberts, B. (1998). ESS systems evolve to serve virtual offices. $H R$ Magazine, 43(13), 24-29.

Rogers, M. (2000). Homegrown goodness. Employee Benefit News, 14(6), 1-4.

Starcke, A.M. (1997). HR Self-service, beyond the technology. $H R$ Magazine, 42(9), 37-42.

Stedman, C. (1999). SAP to open up R/3 to the web. Computerworld, 33(18), 8-9.

Townsend, A. M. \& Hendrickson. A. R. (1996). Recasting HRIS as an information resource, HR Magazine, 41(2), 91-94.

Ulrich, D. (1997). Judge me more by my future than by my past. Human Resource Management, 36(1), 5-8. 